\title{
The Measurement of the Number of Light Neutrino Species at LEP
}

\author{
Salvatore Mele \\ CERN, CH-1211 Geneva 23, Switzerland \\ salvatore.mele@cern.ch
}

\begin{abstract}
Within weeks of the start of the data taking at the LEP accelerator, the ALEPH, DELPHI, L3 and OPAL experiments were able to confirm the existence of just three light neutrino species. This measurement relies on the Standard Model relation between the 'invisible' width of the Z-boson and the cross-sections for Z-boson production and subsequent decay into hadrons.

The full data sample collected by the experiments at and around the Z-boson resonance allows a high-precision measurement of the number of light neutrino species as $2.9840 \pm 0.0082$. The uncertainty is mostly due to the understanding of the low-angle Bhabha scattering process used to determine the experimental luminosity.

This result is independently confirmed by the elegant direct observation of the $\mathrm{e}^{-} \mathrm{e}^{+} \rightarrow \nu \bar{\nu} \gamma$ process, through the detection of an initial-state-radiation photon in otherwise empty detectors.

This result confirms expectations from the existence of three charged leptons species, and contributes to the fields of astrophysics and cosmology. Alongside other LEP achievements, the precision of this result is a testament to the global cooperation underpinning CERN's fourth decade. LEP saw the onset of large-scale collaboration across experiments totaling over 2000 scientists, together with a strong partnership within the wider high-energy physics community: from accelerator operations to the understanding of theoretical processes.
\end{abstract}

\section{Introduction}

The inception, design and approval of the LEP program at CERN, and the subsequent monumental construction of the accelerator and the detectors, represents a watershed in the history of the laboratory. The scientific, organizational and sociological challenges are described first hand in Ref. 1. The largest scientific instrument ever built, LEP was designed to push the frontiers of knowledge and understand the Standard Model of the electroweak interactions, with high-precision measurements of the properties of the recently discovered $\mathrm{Z}$ and $\mathrm{W}$ bosons.

One in five Z bosons produced at LEP decays into a "light neutrino", that is a neutrino whose mass is less than half the Z-boson mass. The Standard Model relation between this decay width and the cross-section for Z-boson production and subsequent decay into hadrons makes it possible to infer the number of light neutrino species, $N_{\nu}{ }^{2}$ 
Table 1 Early measurements of the number of light neutrino species by each of the four LEP experiments, and their average. Uncertainties are dominated by the scale of the observed Z-boson production cross-section.

\begin{tabular}{lcc}
\hline Experiment & $N_{\nu}$ & Reference \\
\hline ALEPH & $3.27 \pm 0.30$ & 4 \\
DELPHI & $2.4 \pm 0.6$ & 5 \\
L3 & $3.42 \pm 0.48$ & 6 \\
OPAL & $3.1 \pm 0.4$ & 7 \\
Average & $3.10 \pm 0.04$ & 8 \\
\hline
\end{tabular}

The first beams circulated in the LEP accelerator on 14th July 1989. The first data-taking campaign was at centre-of-mass energies around $90 \mathrm{GeV}$, compatible with the mass ${ }^{\mathrm{a}}$ of the $\mathrm{Z}$ boson measured by the UA1 and UA2 experiments. ${ }^{3}$ By mid October 1989, the four LEP experiments, ALEPH, DELPHI, L3 and OPAL, had already published their first articles describing the Z boson properties. ${ }^{4-7}$ As listed in Table 1, these early observations already allowed to constrain $N_{\nu}$ to be around 3 .

This article describes in details the measurement of $N_{\nu}$ in the context of the LEP high-precision physics program. Beyond the physics achievement, this measurement gives an insight on what made the LEP program so successful: a unique combination of exceptional accelerator performance, creative technological achievements in building and operating the detectors, and unprecedented cooperation with the theoretical physics community. These aspects set the scene for turning an important page in the history of CERN, then in its fourth decade. Such an enhanced culture of collaboration would usher the LHC era in CERN's fifth and sixth decades, as a worldwide hub of cooperation and creativity.

The structure of this article is the following. After this introduction, Section 2 recalls the principles of the indirect measurement of $N_{\nu}$, including some concepts of the Standard Model of the electroweak interactions. Section 3 describes the experimental approach, including a broad-brush description of the LEP detectors, and presents the results of the measurement and a discussion of the uncertainties. Section 4 highlights a complementary direct measurement of $N_{\nu}$ through the detection of spectacular events with a single photon in otherwise empty detectors. Section 5 offers some concluding considerations.

\section{Theoretical Principles}

A cornerstone of the LEP physics program is the study of the Z-boson "lineshape". This encompasses the measurement of parameters of the Standard Model of the

${ }^{\text {a We assume }} h=2 \pi$ and $c=1$, while using the factor 0.389 to convert $\mathrm{GeV}^{2}$ into $\mathrm{mb}^{-1}$. 
electroweak interactions, and the proof of its internal consistency, through the study of physical observables describing Z-boson production and decay. Among these observables, the 'invisible' width of the Z-boson is related to its decay into neutrinos and gives access to $N_{\nu}$. This section presents the physical observables leading to the measurement of $N_{\nu}$ and some key theoretical assumptions.

\subsection{The width of the $Z$ boson}

The width of the $\mathrm{Z}$ boson is defined as

$$
\Gamma_{\mathrm{Z}}=\Gamma_{\mathrm{ee}}+\Gamma_{\mu \mu}+\Gamma_{\tau \tau}+\Gamma_{\mathrm{had}}+N_{\nu} \Gamma_{\nu \nu}
$$

where the first three terms are the widths of decays into electrons, muons and taus, respectively. $\Gamma_{\text {had }}$ is the sum of the widths of decays into $\mathrm{u}, \mathrm{d}, \mathrm{s}, \mathrm{c}$ and b quarks and $\Gamma_{\nu \nu}$ the width of decays into neutrinos. The simultaneous measurement of $\Gamma_{\mathrm{Z}}$, and of observables related to the hadronic and leptonic widths of the $\mathrm{Z}$ boson, allows one to determine $N_{\nu}{ }^{2}$

The partial decay widths of the $\mathrm{Z}$ boson into each fermion pair are related to the Z-boson couplings and to Standard Model parameters as: ${ }^{9-11}$

$$
\Gamma_{\mathrm{f} \overline{\mathrm{f}}}=N_{\mathrm{c}}^{\mathrm{f}} \frac{G_{\mathrm{F}} m_{\mathrm{Z}}^{3}}{6 \sqrt{2} \pi}\left(\left|G_{\mathrm{Af}}\right|^{2} R_{\mathrm{Af}}+\left|G_{\mathrm{Vf}}\right|^{2} R_{\mathrm{Vf}}\right)+\Delta_{\mathrm{ew} / \mathrm{QCD}}
$$

where $N_{\mathrm{c}}^{\mathrm{f}}$ is the number of colours (three for quarks and one for leptons), $G_{\mathrm{F}}$ is the Fermi constant determined from muon decay, ${ }^{12} R_{\mathrm{Af}}$ and $R_{\mathrm{Vf}}$ factorise finalstate QED and QCD corrections and contributions from non-zero fermion masses to the axial and vector terms, respectively, $\Delta_{\text {ew } / \mathrm{QCD}}$ accounts for non-factorisable electroweak and QCD corrections, and $G_{\mathrm{Af}}$ and $G_{\mathrm{Vf}}$ are the axial and vector effective couplings of the Z-boson to fermions, written as ${ }^{13}$

$$
\begin{aligned}
& G_{\mathrm{Af}}=\sqrt{R_{\mathrm{f}}} T_{3}^{\mathrm{f}}, \\
& G_{\mathrm{Vf}}=\sqrt{R_{\mathrm{f}}}\left(T_{3}^{\mathrm{f}}-2 Q_{\mathrm{f}} K_{\mathrm{f}} \sin \theta_{\mathrm{W}}\right) .
\end{aligned}
$$

The form factors $R_{\mathrm{f}}$ and $K_{\mathrm{f}}$ absorb the overall scale of the coupling and the on-shell corrections to the electroweak mixing angle, $\theta_{\mathrm{W}}, Q_{\mathrm{f}}$ and $T_{3}^{\mathrm{f}}$ are the electric charge and the third component of weak isospin of the fermion, respectively.

\subsection{Experimental observables}

Four experimental observables describe the total hadronic and leptonic cross section around the Z-boson resonance, and connect $N_{\nu}$ to the Z-boson lineshape:

(1) the mass of the $\mathrm{Z}$ boson, $m_{\mathrm{z}}$;

(2) the width of the $\mathrm{Z}$ boson, $\Gamma_{\mathrm{z}}$; 
(3) the hadronic pole cross-section

$$
\sigma_{\text {had }}^{0}=\frac{12 \pi}{m_{\mathrm{Z}}^{2}} \frac{\Gamma_{\mathrm{ee}} \Gamma_{\mathrm{had}}}{\Gamma_{\mathrm{Z}}^{2}} ;
$$

(4) the ratio of the Z-boson partial decay widths into hadrons and massless leptons, $\Gamma_{l l}$, assuming lepton universality:

$$
R_{l}^{0}=\frac{\Gamma_{\mathrm{had}}}{\Gamma_{l l}}
$$

The non-negligible tau mass is accounted for as $\Gamma_{l l}=\delta_{\tau} \Gamma_{\tau \tau}$, with $\delta_{\tau}=-0.23 \%$. A fifth experimental observable, less important for the $N_{\nu}$ determination, is the lepton forward-backward asymmetry, again assuming lepton universality:

(5) $A_{\mathrm{FB}}^{0,1}$,

which is defined as the asymmetry at the Z-boson pole of the cross-sections for final state leptons emitted in the forward (i.e. the negative-charged lepton 'continuing' along the direction of the incoming electron) or backward direction, according to the general formula $A_{\mathrm{FB}}=\left(\sigma_{\mathrm{F}}-\sigma_{\mathrm{B}}\right)\left(\sigma_{\mathrm{F}}+\sigma_{\mathrm{B}}\right)$.

\subsection{Sensitivity to $N_{\nu}$}

A key experimental observable directly related to $N_{\nu}$ is

$$
R_{\mathrm{inv}}^{0}=\frac{\Gamma_{\mathrm{inv}}}{\Gamma_{l l}}=N_{\nu}\left(\frac{\Gamma_{\nu \nu}}{\Gamma_{l l}}\right) .
$$

The asset of $R_{\mathrm{inv}}^{0}$ is that uncertainties of experimental and theoretical nature are well-controlled in the ration of the Z-boson widths.

The combination of Eqs. (2) and (5) allows to write $R_{\text {inv }}^{0}$ as

$$
R_{\mathrm{inv}}^{0}=\left(\frac{12 \pi R_{l}^{0}}{\sigma_{\mathrm{had}}^{0} m_{\mathrm{Z}}^{2}}\right)^{\frac{1}{2}}-R_{l}^{0}-\left(3+\delta_{\tau}\right),
$$

which expresses, together with Eq. (7), the relation between $N_{\nu}$ and the hadronic pole cross-section. This dependency drives the determination of $N_{\nu}$ and is graphically displayed in Fig. 1, which compares the measured hadron production crosssection around the Z-boson resonance with predictions for two, three and four light neutrino species. The curves in Fig. 1 allow to visualise the enormous statistical sensitivity of LEP data to $N_{\nu}$.

It is important to summarise the assumptions made in describing the dependence of $N_{\nu}$ on the physical observables at LEP: lepton universality holds; Z bosons only decay to known fermions; neutrino masses are negligible; and Z-boson couplings to neutrinos are described by the Standard Model. 


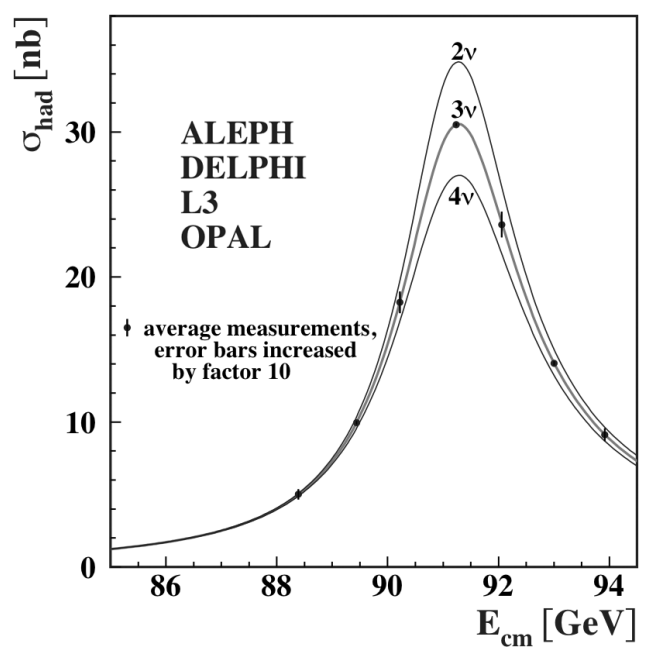

Fig. 1. Measurement of the hadron production cross-section as a function of the LEP centre-ofmass energy around the Z-boson resonance. Combined results from the four LEP experiments are presented. Curves represent the predictions for two, three and four neutrino species. To further convey the high sensitivity of the measurement, uncertainties are magnified tenfold. ${ }^{14}$

\section{Experimental Measurement}

The LEP accelerator and the LEP detectors were unprecedented in their size and complexity. This Section gives a succinct description of how the challenges of highprecision Z-boson detection guided detector design. After recalling the data sample, the measurements of the key observables leading to $N_{\nu}$ are presented, together with the final result, and crucial uncertainties are discussed.

\subsection{Detection of $Z$-boson decays}

The design of the four LEP experiments ${ }^{15-18}$ was optimised to detect Z-boson decays with high efficiency, within the available budgetary, technological and physical constraints. Teams of several hundred scientists, technicians and engineers designed, prototyped, built and assembled sophisticated apparatuses with dimensions exceeding 10 metres in diameter and length, and weighting several thousand tons. While the basic design principles of the detectors were similar, the choices of particular technologies in some sub-detectors were markedly different and would eventually contribute to reduce combined systematical uncertainties.

Figure 2 presents a cut-away three-dimensional view of the four detectors. All are radially and forward-backward symmetric. The common part of the design is the succession of sub-detectors, moving outwards from the beam axis: tracking chambers, surrounded by calorimeters and bending magnets, with muon spectrometers as the outmost layer. The exception is the L3 detector where the 

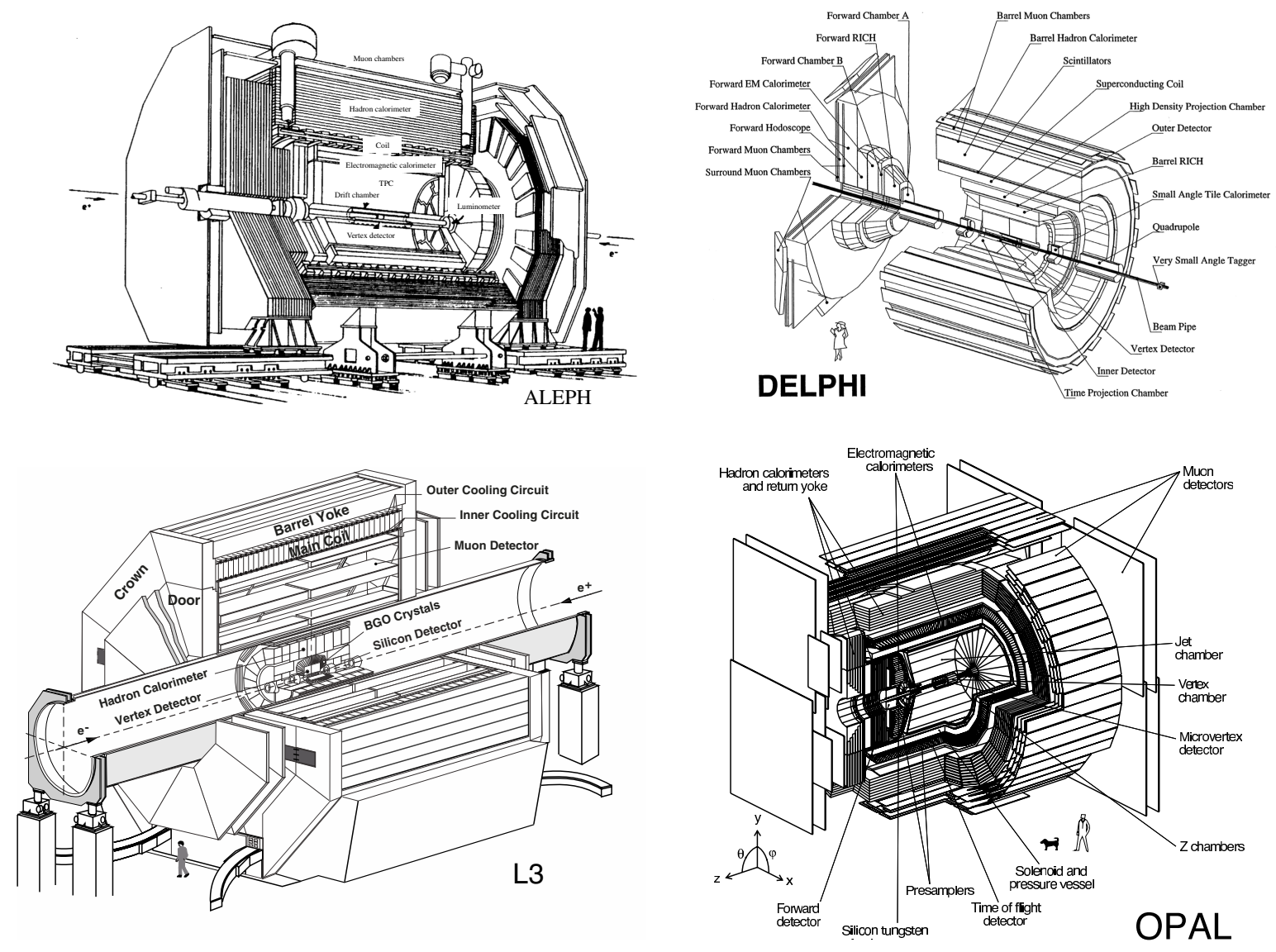

Fig. 2. Cut-away representation of the four LEP detectors: ALEPH, DELPHI, L3 and OPAL. 
entire muon spectrometer is contained in the magnetic field. Some sub-detectors relied on established technology, such as wire chambers for tracking or crystals and scintillator counters for calorimetry, pushing technologies in scale and precision (e.g. the L3 BGO electromagnetic calorimeter, or its high-precision muon spectrometer). Other sub-detector relied on newer technologies, never deployed before on such a large scale (e.g. the ALEPH and DELPHI time-projection chambers, the ALEPH liquid-argon calorimeter and the DELPHI ring imaging Cerenkov detector $\mathrm{RICH})$.

Some examples of the performance of the LEP detectors are the following:

- the transverse momentum resolution of the ALEPH tracking system, $\sigma\left(1 / p_{\mathrm{t}}\right)=0.6 \times 10^{-3} \mathrm{GeV}^{-1} ;^{19}$

- the DELPHI RICH efficiency of $70 \%$ to identify $\mathrm{K}^{ \pm}$with a contamination of $30 \% ;^{20}$

- the energy resolution of the L3 electromagnetic calorimeter $\Delta E / E \approx 1.4 \%$ for $45 \mathrm{GeV}$ electrons; ${ }^{21}$

- the momentum resolution of the L3 muon spectrometer $\Delta p / p \approx 2.5 \%$ for $45 \mathrm{GeV}$ muons. ${ }^{21}$

Figure 3 illustrates the detection principles for Z-boson decays. Hadronic events are identified from a high multiplicity of tracks in the central trackers and energy deposits in the calorimeters, reconstructed in two back-to-back fully-contained jets. Higher jet multiplicity is possible for rarer higher-order QCD processes. Z-boson decays in electron-positron pairs are characterised by two back-to-back tracks in the central trackers, corresponding to high-energy signals in the electromagnetic calorimeters. Z-boson decays into muons have the unique signature of back-to-back tracks in the central trackers, leaving minimum ionising deposits in the hadronic and electromagnetic calorimeters and tracks in the muon chambers. Z-boson decays in tau pairs are more challenging to detect, requiring a combination of missing energy in the detector, low-multiplicity jets, muons or electrons, according to the tau decay channels.

\subsection{Data sample}

The LEP accelerator operated at and around the Z-boson resonance from its commissioning in 1989 through 1995. In 1990 and 1991, energy scans at a spacing of $1 \mathrm{GeV}$ provided a first mapping of the Z-boson resonance. In the following years, high-luminosity data-taking concentrated on the Z-boson resonance, with two additional "off-peak" energy points in 1993 and 1995, 1.8 GeV above and below the Z-boson resonance, to further constrain the Z-boson lineshape. Further details on the LEP accelerator design and performance are given in Ref. 22.

A total of 17 million Z-boson decays were detected by the four experiments. Table 2 provides a breakdown of the integrated luminosity per each experiment and the total number of events detected in the hadronic and leptonic final states. 

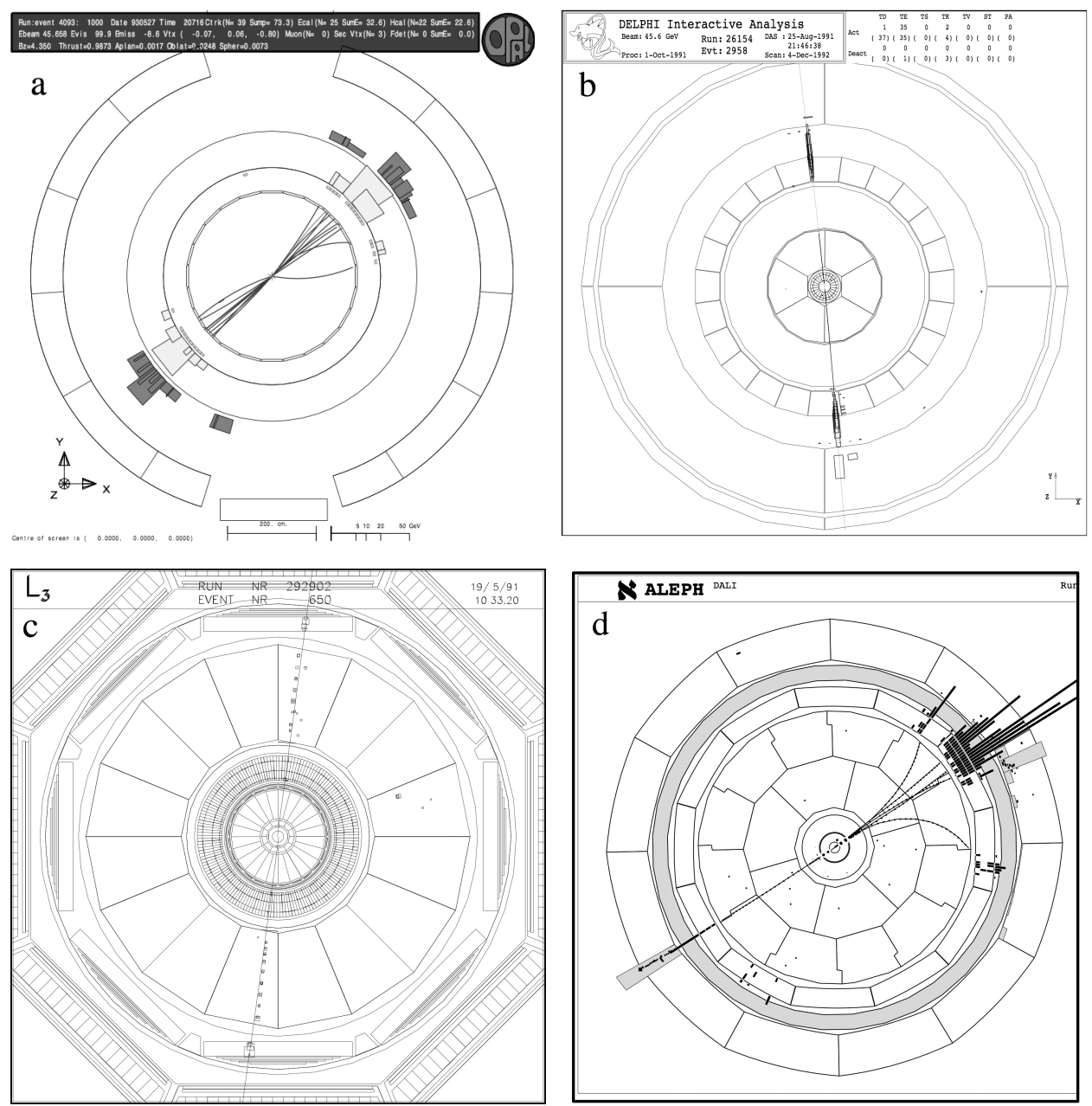

Fig. 3. Event displays of Z-boson decays detected in the four LEP experiments: (a) hadronic decays with the OPAL detector, characterised by two high-multiplicity back-to-back fullycontained jets; (b) electron-positron pairs with DELPHI, with two back-to-back tracks in the central tracker, and two energy deposits in the electromagnetic calorimeter, with energies close to the beam energy; (c) muon pairs with L3, with tracks in the muon chambers (mostly outside the image), minimum ionizing deposits in the hadronic and electromagnetic calorimeters and corresponding tracks in the central chambers, time-of-flight detectors assure such tracks are originating from the collision vertex and not from cosmic rays; (d) tau pairs with ALEPH, in this case with an electron (track and calorimeter deposit) detected in the hemisphere opposite a collimated, low-multiplicity jet, with overall missing energy. In all images the beam axis is perpendicular to the page. 
Table 2 Centre-of-mass energy and luminosity delivered to each experiment, and total numbers of events collected by the four experiments in the hadronic and leptonic decay modes. Due to the low integrated luminosity and relative control of the experimental conditions, the 1989 data sample is not used in the study of the Z-boson lineshape.

\begin{tabular}{lcccc}
\hline Year & $\begin{array}{c}\text { Centre-of-mass } \\
\text { energy } \\
{[\mathrm{GeV}]}\end{array}$ & $\begin{array}{c}\text { Integrated } \\
\text { luminosity/experiment } \\
{\left[\mathrm{pb}^{-1}\right]}\end{array}$ & $\begin{array}{c}\text { Total detected } \\
\text { hadronic events } \\
{\left[\times 10^{3}\right]}\end{array}$ & $\begin{array}{c}\text { Total detected } \\
\text { leptonic events } \\
{\left[\times 10^{3}\right]}\end{array}$ \\
\hline $1990 / 91$ & $88.2-94.2$ & 27.5 & 1660 & 186 \\
1992 & 91.3 & 28.6 & 2741 & 294 \\
1993 & $89.4,91.2,93.0$ & 40.0 & 2607 & 296 \\
1994 & 91.2 & 64.5 & 5910 & 657 \\
1995 & $89.4,91.3,93.0$ & 39.8 & 2579 & 291 \\
\hline
\end{tabular}

\subsection{Measurement of cross-sections and asymmetries}

In each final state of Z-boson decays, cross-sections are measured as $\sigma_{\text {tot }}=\left(N_{\mathrm{s}}-\right.$ $\left.N_{\mathrm{b}}\right) / \varepsilon \mathcal{L}$, where $N_{\mathrm{s}}$ is the number of selected events, $N_{\mathrm{b}}$ is the number of events expected from background processes, $\varepsilon$ is the selection efficiency, which include geometrical acceptance, and $\mathcal{L}$ is the integrated luminosity. The LEP experiments derive $N_{\mathrm{b}}$ and $\varepsilon$ from Monte Carlo generators describing the kinematics of both the Z-boson production and decay and of background processes. Events produced with those generators are passed through detailed simulations of the detectors and the same software used to reconstruct collision events. These workflows are crosschecked by using data and refined through the years to give extremely accurate simulation of the detectors.

Asymmetries for each final state are measured as $A_{\mathrm{FB}}=\left(N_{\mathrm{F}}-N_{\mathrm{B}}\right) /$ $\left(N_{\mathrm{F}}+N_{\mathrm{B}}\right)$, where $N_{\mathrm{F}}$ and $N_{\mathrm{B}}$ are event counts for negatively changed leptons 'continuing' along the direction of the incoming electron, or emitted 'backwards', respectively.

The large statistical sample of Z-boson decays collected at LEP results in low statistical uncertainties in the cross-section determinations for each experiment, around 0.5 per mille in the hadronic channel and 2.5 per mille in the leptonic channels. Experiment-dependent systematic uncertainties are mostly due to the calculation of efficiencies and acceptances and the selection procedures, as estimated from data and Monte Carlo simulations. These vary between 0.4 and 0.7 per mille in the hadronic channel and 1 to 7 per mille in the leptonic channel, with the higher value corresponding to tau pairs. For asymmetries, experiment-dependent systematic uncertainties have absolute values between 0.0005 and 0.0030 , with the higher value corresponding to tau pairs. Statistical uncertainties are between two and five times larger than the systematic uncertainties. ${ }^{14,}{ }^{23-26}$

Systematic uncertainties on cross-sections and asymmetries which are common across experiments are irreducible. The main sources are: the LEP energy calibration; ${ }^{22}$ the use of the same Monte Carlo generators to simulate signal 
and background processes; theoretical uncertainties on the parametrisation of Standard Model observables, contributions to the electron-positron final states, and the overall QED final-state corrections. The most important source of common systematical uncertainties affects the determination of luminosity, as discussed in the next section.

\subsection{Measurement of luminosity}

As presented in Eqs. (7) and (8), and in Fig. 1, $N_{\nu}$ depends strongly on the scale of the hadronic cross-section. As detectors are well understood and the large event counts limit statistical uncertainties, the $N_{\nu}$ precision depends on the accuracy of the luminosity measurement. LEP experiments relied on the detection of low-angle Bhabha scattering events for the measurement of instantaneous luminosity. ${ }^{27}$ The advantages of this process is a high cross-section and therefore a negligible statistical uncertainty, as well as a low contribution from Z-boson production itself.

Pairs of dedicated calorimeters, completed with tracking devices, were installed close to the LEP beam pipe, in the forward and backward low-angle regions, typically between 30 and $50 \mathrm{mrad}$ from the beam axis. Delicate to operate, these instruments had to be protected from hazardous conditions while beams were manipulated in the machine before stable collisions, and would then count coincidence of energy deposits in the forward and backward regions, originated by charged particles and compatible with the beam energy: the typical signature of Bhabha scattering. Event counts yield a detailed record of the instantaneous luminosity conditions and then allow to extract the total integrated luminosity. Experimentdependent, systematic uncertainties for the determination of the luminosity are well controlled, in the range $0.03-0.09 \%$.

All experiment relied on the same Monte Carlo generator and state-of-the art theoretical calculations to estimate the accepted low-angle Bhabha scattering cross-section, and derive the luminosity. ${ }^{28}$ After intense effort in improving these calculations, a residual theoretical uncertainty of $0.061 \%$ remains, mostly originating from vacuum polarisation, higher-order corrections and the production of light fermion pairs. ${ }^{29}$ The way the luminosity uncertainty has been reduced over the LEP data-taking campaign tells a success story of highly sophisticated experimental techniques moving in lockstep with dedicated efforts by the theory community to push the understanding of the calculation of low-angle Bhabha scattering.

The LEP-wide combination of cross-sections and asymmetries, in addition to the obvious statistical advantages, allows to reduce several uncorrelated systematic uncertainties of experimental origin. At the same time, the theoretical uncertainty on the determination of the luminosity uncertainty is common to all experiments, and therefore irreducible. It contributes as much as a half of the uncertainty on the hadronic pole cross-section determination and dominates the systematic uncertainty on the determination of the $N_{\nu}$, as discussed in the following sections. 


\subsection{Results}

Each LEP experiment extracted cross-sections and asymmetries in the hadronic and leptonic final states at different energy points, corresponding to about 200 individual measurements. These allowed a precise description of the Z-boson lineshape and the corresponding extraction of parameters of the Standard Model. ${ }^{23-26}$

An additional, through then unprecedented, collaborative efforts across the experiments led to the establishment of the LEP ElectroWeak Working Group. ${ }^{30}$ The Group had the mandate to devise and arrange the combination of the Z-boson lineshape measurements across the experiments and thus obtain a considerable reduction of uncertainties, both of a statistical and systematic nature. Each experiments provided results in agreed-upon formats, with full correlation matrices. The LEP ElectroWeak Working Group combined ${ }^{14}$ all inputs to both determine the Z-boson lineshape observables with a much higher precision than allowed by each individual experiment statistical sample and check the overall consistency of the results and their implication for the understanding of the Standard Model. ${ }^{22}$ Table 3 presents combined results for the observables introduced in Section 2.2, in the hypothesis of lepton universality. The combination shows the compatibility of results across the experiments, with a goodness-of-fit of $\chi^{2} /$ d.o.f. $=36.5 / 31$.

It is important to remark that the lepton universality hypothesis is tested in the entire LEP data sample by measuring the rations of the $\mathrm{Z}$ boson partial decay widths as $\Gamma_{\mu \mu} / \Gamma_{e e}=1.0009 \pm 0.0028$ and $\Gamma_{\tau \tau} / \Gamma_{e e}=1.0019 \pm 0.0032 .^{14}$

Using Eqs. (7) and (8) and the Standard Model value for the ratio of the Z-boson widths to neutrinos and leptons ${ }^{14}$

$$
\left(\Gamma_{\nu \nu} / \Gamma_{l l}\right)_{\mathrm{SM}}=1.99125 \pm 0.00083,
$$

the number of light neutrino species is determined as:

$$
N_{\nu}=2.9840 \pm 0.0082 \text {. }
$$

It is important to recall the four key assumptions leading to this result:

- lepton universality holds;

- no other Z-boson decays exist beyond those to known fermions;

Table 3 Combined LEP results, and their correlation for key observables (Section 2.1). ${ }^{14}$

\begin{tabular}{ccccccc}
\hline & & \multicolumn{5}{c}{ Correlations } \\
\cline { 3 - 7 } Observable & Combined LEP measurement & $m_{\mathrm{z}}$ & $\Gamma_{\mathrm{z}}$ & $\sigma_{\mathrm{had}}^{0}$ & $R_{l}^{0}$ & $A_{\mathrm{FB}}^{0,1}$ \\
\hline$m_{\mathrm{z}}$ & $91.1875 \pm 0.021 \mathrm{GeV}$ & 1.000 & & & & \\
$\Gamma_{\mathrm{z}}$ & $2.4952 \pm 0.0023 \mathrm{GeV}$ & -0.023 & 1.000 & & & \\
$\sigma_{\mathrm{had}}^{0}$ & $41.540 \pm 0.037 \mathrm{nb}$ & -0.045 & -0.297 & 1.000 & & 1.000 \\
$R_{l}^{0}$ & $20.767 \pm 0.025$ & 0.033 & 0.004 & 0.183 & 0.000 \\
$A_{\mathrm{FB}}^{0,1}$ & $0.0171 \pm 0.0010$ & 0.055 & 0.033 & 0.006 & -0.056 & 1.000 \\
\hline
\end{tabular}


- neutrino masses are negligible;

- Z-boson couplings to neutrinos are described by the Standard Model.

\subsection{Uncertainties}

The uncertainty on $N_{\nu}$ is less the three per mille. It is decomposed as the sum in quadrature of three parts: ${ }^{14}$

$$
\delta N_{\nu} \sim 10.5 \frac{\delta n_{\text {had }}}{n_{\text {had }}} \oplus 3.0 \frac{\delta n_{\text {lep }}}{n_{\text {lep }}} \oplus 7.5 \frac{\delta \mathcal{L}}{\mathcal{L}} .
$$

The first two are related to uncertainties on the number of events selected for the measurement of cross-section and asymmetries in the hadronic and leptonic channels, respectively. The third term parametrises uncertainties on the scale of the cross-sections deriving from the uncertainties on the luminosity measurement.

The largest contribution to the uncertainty on the luminosity measurement is the theoretical uncertainty $(0.061 \%)$ discussed in Section 3.4. This uncertainty alone results in an uncertainty on $N_{\nu}$ of 0.0046 , accounting for more than half of the total uncertainty on $N_{\nu}$.

\section{Direct Measurement of $N_{\nu}$}

The LEP experiments pursued an alternative and elegant measurement of $N_{\nu}$ by detecting events with a single visible photon as a signature of the $\mathrm{e}^{-} \mathrm{e}^{+} \rightarrow \nu \bar{\nu} \gamma$ process. ${ }^{2}$ At the Z-boson resonance, this final state is mostly due to the initialstate radiation of a low-angle photon, with a steeply falling energy spectrum, with a Z boson decaying into neutrinos. Contributions from the t-channel exchange of a virtual $\mathrm{W}$ boson are small.

At the Z-boson resonance, the cross-section of the $\mathrm{e}^{-} \mathrm{e}^{+} \rightarrow \nu \bar{\nu} \gamma$ process can be written $^{31}$ as

$$
\sigma_{\nu \nu \gamma}^{0}(s)=\frac{12 \pi}{m_{\mathrm{Z}}^{2}} \frac{s \Gamma_{\mathrm{ee}} N_{\nu} \Gamma_{\nu \nu}}{\left(s-m_{\mathrm{Z}}^{2}\right)+s^{2} \Gamma_{\mathrm{Z}}^{2} / m_{\mathrm{Z}}^{2}}+\mathrm{W} \text {-boson exchange terms }
$$

which is mostly proportional to $N_{\nu}$. A careful measurement of the cross-section of the process with the control of the residual background sources and the overall acceptance allows to extract $N_{\nu}$. This cross-section is considerably lower than the Z-boson resonance. The statistical accuracy of the direct measurement of $N_{\nu}$ is therefore over an order of magnitude inferior than the indirect measurement. At the same time, the direct measurement does not rely on the assumption that $\mathrm{Z}$ bosons only decay to known fermions. Possible decays into visible 'exotic' particles, conflated within other visible channels and in particular hadronic final states, could in principle alter the Z-boson lineshape and yield an incorrect measurement of $N_{\nu}$.

The key experimental challenge of the direct measurement is to detect events with a single photon and no other activity in the detector. On the one hand, the 
cross-section is larger, and therefore the measurement more sensitive, the lower the energy of the photon and the closest the photon is to the beam axis. On the other hand, these exact conditions make both photon detection more complex and experimental backgrounds harder to control. The four LEP experiments devised sophisticated analysis chains and in some cases even dedicated trigger systems to record these "single photon" events (e.g. the one described in Ref. 32). Around 2500 single photon events, with background subtracted, were collectively detected by the four experiments at the Z-boson resonance, with different energy thresholds and fiducial volumes, as summarised in Table 4, which also details data samples and the signal-over-background ratios.

Figure 4 presents an example of the measured cross-section as a function of the centre-of-mass energy and its dependency on $N_{\nu}$. Fits to the theoretical modeling of the cross-section, with the assumption of Standard Model coupling of the Z-boson to neutrinos, yield the individual direct measurements of $N_{\nu}$ listed in Table 4 . These results can be combined $\mathrm{as}^{37}$ :

$$
N_{\nu}=3.00 \pm 0.08
$$

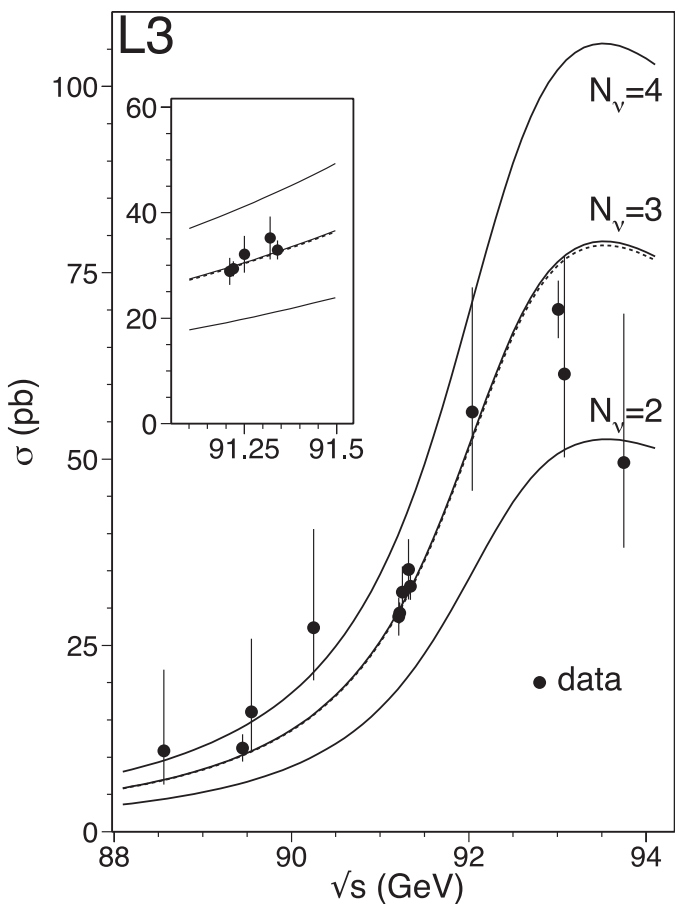

Fig. 4. Cross-section measured by the L3 experiment for the $\mathrm{e}^{-} \mathrm{e}^{+} \rightarrow \nu \bar{\nu} \gamma$ process around the Z-boson resonance as a function of the centre-of-mass energy. The lower limit for the photon energy is $1 \mathrm{GeV}$, and the fiducial volume $\left|\cos \theta_{\gamma}\right|<0.71$. Theoretical predictions for two, three or four light neutrinos species are also shown. The dashed line represents a fit to the data points. ${ }^{35}$ 
Table 4 Integrated luminosity, $\mathcal{L}$, photon energy threshold, $E_{\gamma}$, and fiducial volume, $\left|\cos \theta_{\gamma}\right|$, for the four LEP experiments' analyses of single-photon events around the Z-boson resonance. The signal over background ratios, s/b, are also given, together with each experiment direct measurement of $N_{\nu}$ and their average. The first uncertainties are statistical and the second systematic.

\begin{tabular}{lcccccc}
\hline & $\mathcal{L}$ & $\begin{array}{c}E_{\gamma}> \\
{[\mathrm{GeV}]}\end{array}$ & $\left|\cos \theta_{\gamma}\right|<$ & $\mathrm{s} / \mathrm{b}$ & $N_{\nu}$ & Reference \\
\hline ALEPH & 15.7 & 1.5 & 0.74 & 1.8 & $2.68 \pm 0.20 \pm 0.20$ & 33 \\
DELPHI & 67.6 & 3.0 & 0.70 & 2.7 & $2.89 \pm 0.32 \pm 0.19$ & 34 \\
L3 & 99.9 & 1.0 & 0.71 & 6.0 & $2.98 \pm 0.07 \pm 0.07$ & 35 \\
OPAL & 40.5 & 1.75 & 0.70 & 11.0 & $3.23 \pm 0.16 \pm 0.10$ & 36 \\
& & & & Average & $3.00 \pm 0.08$ & 37 \\
\hline
\end{tabular}

Table 5 Direct measurement of $N_{\nu}$ at centre-of-mass energies, $\sqrt{s}$, above the Z-boson resonance. Each experiments investigated different observables to extract $N_{\nu}$.

\begin{tabular}{lclcr}
\hline Experiment & $\sqrt{s}[\mathrm{GeV}]$ & Observable(s) & $N_{\nu}$ & Reference \\
\hline ALEPH & $189-207$ & Missing mass, $\theta_{\gamma}$ & $2.86 \pm 0.09$ (stat.+syst.) & 41 \\
DELPHI & $130-209$ & Cross section & $2.84 \pm 0.10$ (stat.) \pm 0.14 (syst.) & 42 \\
L3 & $130-209$ & Recoil mass, $\theta_{\gamma}$ & $2.95 \pm 0.08$ (stat.) \pm 0.03 & 43 \\
OPAL & $130-189$ & $E_{\gamma}$ & (syst.) \pm 0.03 (th.) & 44 \\
Average (including lower energies) & $3.27 \pm 0.30$ (stat.+syst.) & 37 \\
\hline
\end{tabular}

The LEP experiments repeated this measurements at centre-of-mass energies above the Z-boson resonance. At these higher energies, from $130 \mathrm{GeV}$ to $209 \mathrm{GeV}$, the single-photon energy spectrum exhibits two distinct features. The first feature is a steeply falling behavior similar to that observed at the Z-boson resonance, mostly due to the initial-state radiation of a photon accompanying the t-channel production of a neutrino-antineutrino pair through the exchange of a virtual $\mathrm{W}$ boson. The second feature is a peak at the energy corresponding to the difference between the centre-of-mass energy and the Z-boson mass. This structure corresponds to the radiation in the initial state of a photon of the energy needed to lower the centre-ofmass energy back to the $\mathrm{Z}$-boson resonance, with a $\mathrm{Z}$ boson decaying into neutrinos. Monte Carlo simulations of these processes ${ }^{38-40}$ allow to model the dependence of the photon energy spectrum, and its polar angle, on $N_{\nu}$.

The four experiments collectively detected about 6200 single photon events above the Z-boson resonance, with relatively low background. The study of various observables allows to extract $N_{\nu}$, with the results summarised in Table 5 . Including lower-energy data, the combined result for the direct determination of the number of light neutrino species across all LEP energies is: ${ }^{37}$

$$
N_{\nu}=2.92 \pm 0.05
$$




\section{Conclusions}

In 1989, within the first few weeks of data taking at LEP, the ALEPH, DELPHI, L3 and OPAL collaborations reported the number of light neutrino species to be around three. This is a remarkable achievement which bears witness to the performance of the LEP accelerator, the early understanding of detectors, and the overall planning of the LEP physics program: the most complex CERN had seen in its first four decades. It would take five more years of data-taking, and about a decade more to develop sophisticated analysis techniques to combine results across the LEP experiments, for the final determination of the number of light neutrino species to be published as: ${ }^{14}$

$$
N_{\nu}=2.9840 \pm 0.0082 .
$$

The dominating uncertainty is the theoretical control of the low-angle Bhabha scattering process used to determine the experimental luminosity. This result relies on four important assumptions: that lepton universality holds; that Z bosons only decay to known fermions; that neutrino masses are negligible; and finally that Zboson couplings to neutrinos are as described by the Standard Model. The direct measurement of the $\mathrm{e}^{-} \mathrm{e}^{+} \rightarrow \nu \bar{\nu} \gamma$ process, at the Z-boson resonance and at higher centre-of-mass energies up to $209 \mathrm{GeV}$, allows an independent verification, obtaining a value $N_{\nu}=2.92 \pm 0.05$.

This result stands out as one of the legacies of the LEP physics program. It ruled out for the first time the existence of a fourth generation, and poses stringent limits on theoretical models relevant in astrophysics and cosmology. The high precision of the result further constrains the existence of exotic particles in Z-boson decays. Beyond the tremendous physical importance, the impressive precision of the measurement of the number of light neutrino species at LEP, and the overall determination of the parameters of the Standard Model and the proof of its internal consistency, ${ }^{22}$ mark a turning point in the history of CERN as an example of scientific cooperation.

The LEP detectors where the first to be built by truly worldwide collaborations, with large contingents of scientists from the United States and Asia participating to a CERN program. Unprecedented in size, the LEP collaborations were the mold for the true globalisation of particle physics as an enterprise, and of CERN as a laboratory, which ushered the LHC era over the two most recent decades in CERN's history. This example of global scientific collaboration has captured worldwide attention, and imagination, at the time of the first LHC discoveries. It is more than an anecdote, but rather a proof of how scientific cooperation is indispensible to extend human knowledge, that the scientific publication describing the highprecision measurements at $\mathrm{LEP}^{14}$ was signed by over 2500 authors, the first ever published article to do so. ${ }^{\mathrm{b}}$

\footnotetext{
${ }^{\mathrm{b}}$ Contrary to what is sometimes heard, the first published article with more than 1000 authors is not on high-energy physics, but about a large-scale Japanese medical study. ${ }^{45,} 46$
} 
The LEP era transformed CERN, with large and crucial contributions from scientists of the then Soviet Union and countries from Eastern Europe, alongside scientists from the United States and Western Europe. This process enshrined the crucial role of CERN as an ambassador of 'Science for Peace', recently recognised by the United Nations in granting CERN observer status at its General Assembly.

On the one hand, the precise determination of the number of light neutrino species is of fundamental importance for our understanding of the Universe. On the other hand, the decade-long global cooperative effort to achieve this result, through the ingenuity and creativity of thousands of dedicated individuals, is part of our collective legacy as the human species.

\section{References}

1. H. Schopper, LEP - The Lord of the Collider Rings at CERN (Springer, 2009).

2. G. Barbiellini et al., Neutrino counting, in Proceedings of the Workshop on Z Physics at LEP, CERN, Switzerland (Sept. 1989), pp. 129-170.

3. C. Rubbia, The discovery of the $\mathrm{W}$ and $\mathrm{Z}$ particles, in 60 Years of CERN Experiments and Discoveries. (World Scientific, 2015).

4. D. Decamp et al. (ALEPH Collaboration), A precise determination of the number of families with light neutrinos and of the z-boson partial widths, Phys. Lett. B 231, 519-529 (1989).

5. P. Aarnio et al. (DELPHI Collaboration), Measurement of the mass and width of the $\mathrm{Z}^{0}$-particle from multihadronic final states produced in $\mathrm{e}^{+} \mathrm{e}^{-}$annihilations, Phys. Lett. B 231, 539-547 (1989).

6. B. Adeva et al. (L3 Collaboration), A determination of the properties of the neutral intermediate vector boson $\mathrm{Z}^{0}$, Phys. Lett. B 231, 509-518 (1989).

7. M. Z. Akrawy et al. (OPAL Collaboration), A precise determination of the number of families with light neutrinos and of the Z-boson partial widths, Phys. Lett. B 231, 530-538 (1989).

8. J. J. Hernandez et al. (Particle Data Group), Review of particle properties, Phys. Lett. B 239, VI.22-VI.23 (1990).

9. K. Chetyrkin et al., QCD corrections to the $\mathrm{e}^{+} \mathrm{e}^{-}$cross section and the Z-boson decay rate. In Reports of the working group on precision calculations for the $Z$ resonance, CERN, Switzerland (Mar. 1993), pp. 175-264.

10. A. Czarnecki and J. Kuhn, Nonfactorizable QCD and electroweak corrections to the hadronic Z boson decay rate, Phys. Rev. Lett. 77, 3955-3958 (1996).

11. R. Harlander et al., Complete corrections of $\mathrm{O}\left(\alpha \alpha_{s}\right)$ to the decay of the Z-boson into bottom quarks, Phys. Lett. B 426, 125-132 (1998).

12. K. Olive et al. (Particle Data Group), Review of Particle Physics, Chin. Phys. C 38, i-1676 (2014).

13. M. Veltman, Limit on Mass Differences in the Weinberg Model, Nucl. Phys. B 123, 89-99 (1977).

14. S. Schael et al. (ALEPH, DELPHI, L3, OPAL and SLD Collaborations and the LEP Electroweak Working Group, SLD Electroweak Group and SLD Heavy Flavour 
Group Collaborations), Precision electroweak measurements on the Z Resonance, Phys. Rept. 427, 257-454 (2006).

15. D. Decamp et al. (ALEPH Collaboration), ALEPH: A detector for electron-positron annihilations at LEP, Nucl. Instrum. Meth. A 294, 121-178 (1990).

16. P. Aarnio et al. (DELPHI Collaboration), The DELPHI detector at LEP, Nucl. Instrum. Meth. A 303, 233-276 (1991).

17. B. Adeva et al. (L3 Collaboration), The construction of the L3 experiment, Nucl. Instrum. Meth. A 289, 35-102 (1990).

18. K. Ahmet et al. (OPAL Collaboration), The OPAL detector at LEP, Nucl. Instrum. Meth. A 303, 275-319 (1991).

19. D. Busculic et al. (ALEPH Collaboration), Performance of the ALEPH detector at LEP, Nucl. Instrum. Meth. A 360, 481-506 (1995).

20. P. Abreu et al. (DELPHI Collaboration), Performance of the DELPHI detector, Nucl. Instrum. Meth. A 378, 57-100 (1996).

21. O. Adriani et al. (L3 Collaboration), Results from the L3 experiment at LEP, Phys. Rept. 236, 1-146 (1993).

22. W. de Boer, Precision Experiments at LEP, in 60 Years of CERN Experiments and Discovery (World Scientific, 2015).

23. R. Barate et al. (ALEPH Collaboration), Measurement of the $\mathrm{Z}$ resonance parameters at LEP, Eur. Phys. J. C 14, 1-50 (2000).

24. P. Abreu et al. (DELPHI Collaboration), Cross-sections and leptonic forward backward asymmetries from the Z0 running of LEP, Eur. Phys. J. C 16, 371-405 (2000).

25. M. Acciarri et al. (L3 Collaboration), Measurements of cross-sections and forward backward asymmetries at the $\mathrm{Z}$ resonance and determination of electroweak parameters, Eur. Phys. J. C 16, 1-40 (2000).

26. G. Abbiendi et al. (OPAL Collaboration), Precise determination of the $\mathrm{Z}$ resonance parameters at LEP: 'Zedometry', Eur. Phys. J. C 19, 587-651 (2001).

27. G. M. Dallavalle, Review of precision determinations of the accelerator luminosity in LEP experiments, Acta Phys. Pol. B 28, 901-923 (1997).

28. S. Jadach et al., Upgrade of the Monte Carlo program BHLUMI for Bhabha scattering at low angles to version 4.04, Comput. Phys. Commun. 102, 229-251 (1997).

29. B. Ward et al., New results on the theoretical precision of the LEP/SLC luminosity, Phys. Lett. B 450, 262-266 (1999).

30. LEP Electroweak Working Group, http://lepewwg.web.cern.ch/LEPEWWG/, last accessed February $6^{\text {th }}, 2015$.

31. O. Nicrosini and L. Trentadue, Structure Function Approach to the Neutrino Counting Problem, Nucl. Phys. B 318, 1-21 (1989).

32. R. Bizarri et al., The First level energy trigger of the L3 experiment: Description of the hardware, Nucl. Instrum. Meth. A 317, 463-473 (1992).

33. D. Buskulic et al. (ALEPH Collaboration), A Direct measurement of the invisible width of the Z from single photon counting, Phys. Lett. B 314, 520-534 (1993).

34. P. Abreu et al. (DELPHI Collaboration), Search for new phenomena using single photon events in the DELPHI detector at LEP, Z. Phys. C 74, 577-586 (1997). 
35. M. Acciarri et al. (L3 Collaboration), Determination of the number of light neutrino species from single photon production at LEP, Phys. Lett. B 431, 199-208 (1998).

36. R. Akers et al. (OPAL Collaboration), Measurement of single photon production in $\mathrm{e}^{+} \mathrm{e}^{-}$collisions near the Z0 resonance, Z. Phys. C 65, 47-66 (1995).

37. C. Amsler et al. (Particle Data Group), Review of Particle Physics, Phys. Lett. B 667, 1-1340 (2008).

38. S. Jadach et al., The Precision Monte Carlo event generator K K for two fermion final states in $\mathrm{e}^{+} \mathrm{e}^{-}$collisions, Comput. Phys. Commun. 130, 260-325 (200).

39. S. Jadach et al., The Monte Carlo program KORALZ, version 4.0, for the lepton or quark pair production at LEP / SLC energies, Comput. Phys. Commun. 79, 503-522 (1994).

40. G. Montagna et al., Single photon and multiphoton final states with missing energy at $\mathrm{e}^{+} \mathrm{e}^{-}$colliders, Nucl. Phys. B 541, 31-49 (1999).

41. A. Heister et al. (ALEPH Collaboration), Single photon and multiphoton production in $\mathrm{e}^{+} \mathrm{e}^{-}$collisions at $\sqrt{\mathrm{s}}$ up to $209 \mathrm{GeV}$, Eur. Phys. J. C 28, 1-13 (2003).

42. J. Abdallah et al. (DELPHI Collaboration), Photon events with missing energy in $\mathrm{e}^{+} \mathrm{e}^{-}$collisions at $\sqrt{\mathrm{s}}=130 \mathrm{GeV}$ to $209 \mathrm{GeV}$, Eur. Phys. J. C 38, 395-411 (2005).

43. P. Achard et al. (L3 Collaboration), Single photon and multiphoton events with missing energy in $\mathrm{e}^{+} \mathrm{e}^{-}$collisions at LEP, Phys. Lett. B 587, 16-32 (2004).

44. G. Abbiendi et al. (OPAL Collaboration), Photonic events with missing energy in $\mathrm{e}^{+} \mathrm{e}^{-}$collisions at $\sqrt{\mathrm{s}}=189 \mathrm{GeV}$, Eur. Phys. J. C 18, 253-272 (2000).

45. C. King, Multiauthor Papers: Onward and Upward, in ScienceWatch Newsletter, July 2012, http://archive.sciencewatch.com/newsletter/2012/201207/multiauthor_ papers, last accessed February $6^{\text {th }}, 2015$.

46. H. Nakamura, et al. (MEGA Study Group), Design and baseline characteristics of a study of primary prevention of coronary events with pravastatin among Japanese with mildly elevated cholesterol levels, Circulation J. 68, 860-7 (2004). 